\title{
Relationship Between Confidence, Gender, and Career Choice in Internal Medicine
}

\author{
Katherine Gavinski, MD, MPH', Erin Cleveland, $\mathrm{MD}^{2}$, Aashish K. Didwania, $\mathrm{MD}^{3}$, \\ Joseph M. Feinglass, $P h D^{3}$, and Melanie S. Sulistio, $M D^{\top}$ id
}

'Department of Internal Medicine, Division of Cardiology, University of Texas Southwestern, Dallas, TX, USA; ${ }^{2}$ University of Washington, Seattle, WA, USA; ${ }^{3}$ Northwestern University, Chicago, IL, USA.

BACKGROUND: Understanding factors in internal medicine (IM) resident career choice may reveal important needed interventions for recruitment and diversity in IM primary care and its subspecialties. Self-reported learner confidence is higher in men than in women in certain areas of practicing medicine, but has never been explored as a factor in career choice.

OBJECTIVE: The purpose of this study is to elucidate associations between confidence, gender, and career choice.

DESIGN: IM residents completed a 31-item survey rating confidence in procedural, clinical, and communication skills on a 9-point Likert scale. Residents also reported anticipated career choice and rated influence of factors. Associations between gender and confidence scale scores, gender and career choice, and confidence and career choice were analyzed using $t$ tests, ANOVA, and multiple linear regression controlled for postgraduate year (PGY), institution, and specialty choice.

PARTICIPANTS: 292 IM residents at Northwestern and University of Texas (UT) Southwestern

MAIN MEASURES: Resident gender, self-reported confidence, career choice

KEY RESULTS: Response rate was $79.6 \%(n=292)$, of them 50.3\% women. Overall self-reported confidence increased with training (PGY-1 4.9 (1.1); PGY-2 6.2 (1.0); PGY-3 7.4 (1.0); $p<0.001)$. Men had higher confidence than women (men 6.6 (1.5); women 6.3 (1.4), $p=0.06$ ), with the greatest difference in procedures. High confidence in men was associated with choice of procedural careers, whereas there was no association between confidence and career in women.

Previous Poster Presentations Cleveland E, Gavinski K, Feinglass, JM, Didwania AK, Sulistio M. Relationship Between Confidence, Gender and Career Choice in Internal Medicine Graduate Medical Education. Poster session presented at: Alliance for Academic Internal Medicine; Oct 2018; Orlando, FL.

Gavinski K, Cleveland E, Feinglass JM, Didwania AK, Sulistio M. Relationship Between Confidence, Gender, and Career Choice in Internal Medicine Graduate Medical Education. Poster session presented at: UT Southwestern Celebration of Women in Science and Medicine; Feb 2019; Dallas. TX

Electronic supplementary material The online version of this article (https://doi.org/10.1007/s11606-020-06221-2) contains supplementary material. which is available to authorized users.

Received April 7, 2020

Revised June 28, 2020

Accepted September 4, 2020

Published online September 28, 2020
CONCLUSIONS: This is the first study demonstrating a gender difference in self-reported confidence and career choice. There is a positive correlation in men: higher selfreported confidence with procedural specialties, lower with general internal medicine. Women's self-reported confidence had no association. Further investigation is needed to elucidate causative factors for differences in self-reported confidence by gender, and whether alterations in level of self-reported confidence produce a downstream effect on career choice.

KEY WORDS: gender; confidence; career choice; residency.

Abbreviations
AAMC
GIM
IM
IRB
NW
PGY
UT

Association of American Medical Colleges
general internal medicine
internal medicine
institutional review board
Northwestern
postgraduate year
University of Texas

J Gen Intern Med 36(3):662-7

DOI: $10.1007 / \mathrm{s} 11606-020-06221-2$

(c) Society of General Internal Medicine (This is a U.S. government work and not under copyright protection in the U.S.; foreign copyright protection may apply) 2020

\section{INTRODUCTION}

Multiple factors appear related to ultimate career choice, which are important for recruitment and diversity in internal medicine. ${ }^{1-3}$ Some of these factors are personality traits, 4 prior exposure and educational experiences, economic incentives, mentorship, and perceived work-life balance of the specialties. $^{6-12}$ The association of self-confidence with career choice has not been well-defined. ${ }^{13}$ Discrepancies in selfreported confidence exist between male and female residents. ${ }^{6,13-16}$ Despite having similar grades and test scores in medical school, ${ }^{14}$ male residents report more confidence than their female peers both overall and with regard to procedural competence, but report similar confidence in physician-patient relationships and application of social services. ${ }^{13}$ Despite these differences in self-confidence, external evaluation of residents shows no significant gender difference in clinical skills. ${ }^{17}$ In fact, increased personal estimation of skill has been 
linked to poor performance. ${ }^{18}$ In this study, we queried internal medicine (IM) residents for level of self-reported confidence in a variety of clinical skills, to investigate whether there is an association between confidence and subspecialty choice.

\section{METHODS}

An electronic survey was created based on the competency domains established by the Association of American Medical Colleges (AAMC) to assess clinical self-reported confidence (Appendix 1). The survey consisted of thirty-one tasks representing three skill domains acquired during residency training: clinical, communication, and procedural skills (Appendix 2). Participants rated self-confidence using a rating from 1 to 9 , from "not at all confident" to "very confident." Participants also rated various other factors' influence on ultimate career choice. Career choices were categorized as procedural specialties, non-procedural specialties, and general internal medicine (GIM) (Appendix 3). Procedural specialties included cardiology, gastroenterology, and pulmonary critical care. All other IM specialty services were considered "non-procedural specialties." GIM encompassed both hospital and primary care medicine. Resident gender, postgraduate year, career plans, and ultimate intent for GIM or subspecialty practice were also collected. Institutional review board (IRB) approval was obtained from both UT Southwestern and Northwestern IRB committees.

The survey platform used was Research Electronic Data Capture (REDCap) ${ }^{19}$ The survey was sent to all residents at Northwestern and UT Southwestern IM residencies in 2017. Emails contained a summary of the study intent, description of resident participation, and study disclosures. There were twice as many third-year residents surveyed as other years since two classes of third-year residents received the survey (both departing and newly rising).

Statistical analysis was performed using IBM SPSS Statistics, Version 25 (Armonk, NY).
Confidence ratings of all tasks demonstrated a normal distribution and were summed and divided by 31 to produce an overall 9-point confidence scale (Cronbach's alpha $=0.98$ ) across all items and across confidence subscales (communication, clinical, and procedural, Cronbach's alpha $=0.95,0.97$, and 0.93 , respectively). Mean differences in aggregate and component confidence score values were compared with $t$ tests for dichotomous variables (sex, institution) and ANOVA for multiple categorical variables (PGY-year, career choice). Multiple linear regression was used to test the significance of gender differences in overall confidence scores, controlling for simultaneous associations with postgraduate year, specialty choice, and institution.

\section{RESULTS}

Survey responses were received from 300 residents (79.6\% response rate). Nine were excluded due to largely incomplete responses. Six participants had one confidence item missing, but were included in the final analysis with sample size mean scores adjusted accordingly. Equal numbers of men $(n=145 ; 49.8 \%)$ and women $(n=146$; $50.2 \%)$ responded.

Mean (SD) confidence on all tasks increased with each year of training $(p<0.001)$. Interns had the lowest mean confidence (4.9 (1.1)), compared with second-year $(6.2(1.0))$ and thirdyear residents $(7.4(1.0))$ (Table 1).

Residents were classified by desired future career path: procedural specialties $(n=120)$, non-procedural specialties $(n=111)$, or GIM $(n=54)$. Residents planning to enter a procedural specialty had higher mean confidence $(6.7$ (1.3)) than residents entering non-procedural specialties $(6.3(1.5))$ or GIM (6.2 (1.5)) $(p=0.05)$ (Table 1) and had a higher proportion of men $(n=68 ; 55.7 \%)$. Respondents whose ultimate career choice was GIM had a higher proportion of women $(n=33 ; 61.1 \%)$, though the difference in proportion of men and women going into each career category was not statistically significant $(p=0.11)$.

Table 1 Mean Confidence Scores by Resident Characteristics

\begin{tabular}{lll}
\hline \hline & Sample percent, $\boldsymbol{N = \mathbf { 2 9 1 }}$ & Overall confidence, mean (SD) \\
\hline Postgraduate year (PGY) & & \\
$\quad$ PGY-1 & 25.4 & $4.87(1.07)$ \\
PGY-2 & 27.1 & $6.19(1.04)$ \\
PGY-3 & 47.4 & $7.43(0.95)$ \\
Gender & 50.2 & $6.28(1.44)$ \\
$\quad$ Female & 49.8 & $6.60(1.45)$ \\
$\quad$ Male & 45.7 & $6.54(1.44)$ \\
Institution & 54.3 & $6.36(1.54)$ \\
$\quad$ Northwestern & 18.6 & $6.21(1.52)$ \\
$\quad$ UTSW & 39.5 & $6.29(1.54)$ \\
Specialty choice & 41.9 & $6.68(1.30)$ \\
$\quad$ NIM & &
\end{tabular}

Confidence measured on a 9-point Likert scale 
Table 2 Mean Communication, Clinical, and Procedural Confidence Scores by Specialty Choice and Gender

\begin{tabular}{|c|c|c|c|c|c|c|}
\hline & Communication skills & $p$ value & Clinical skills & $p$ value & Procedural skills & $p$ value \\
\hline \multicolumn{2}{|l|}{ GIM } & \multirow[t]{3}{*}{0.19} & & \multirow[t]{3}{*}{0.02} & & \multirow[t]{3}{*}{0.17} \\
\hline Male, $N=21$ & $6.35(1.18)$ & & $5.65(1.60)$ & & $4.90(2.20)$ & \\
\hline Female, $N=33$ & $6.83(1.36)$ & & $6.64(1.51)$ & & $5.70(2.00)$ & \\
\hline \multicolumn{2}{|c|}{ Non-procedural subspecialty } & \multirow[t]{3}{*}{0.10} & & \multirow[t]{3}{*}{0.08} & & \multirow[t]{3}{*}{0.006} \\
\hline Male, $N=56$ & $6.91(1.35)$ & & $6.61(1.73)$ & & $6.11(2.09)$ & \\
\hline Female, $N=59$ & $6.49(1.35)$ & & $6.07(1.54)$ & & $5.02(2.04)$ & \\
\hline \multicolumn{2}{|c|}{ Procedural subspecialty } & \multirow[t]{3}{*}{0.13} & & \multirow[t]{3}{*}{0.11} & & \multirow[t]{3}{*}{0.03} \\
\hline Male, $N=68$ & $7.07(1.14)$ & & $6.90(1.38)$ & & $6.64(1.62)$ & \\
\hline Female, $N=54$ & $6.75(1.08)$ & & $6.49(1.47)$ & & $5.92(1.96)$ & \\
\hline
\end{tabular}

Confidence measured on a 9-point Likert scale

Female residents reported lower mean confidence scores (6.3 (1.4)) than male residents (6.6 (1.5)), across postgraduate years $(p=0.06)$ (Table 1). Male residents reported higher mean confidence across skill domains in all PG years but this difference was only significant for procedural tasks and for clinical tasks among PGY-3 residents and communication tasks in PGY-1 residents.

Among residents pursuing subspecialty careers, male residents reported higher mean confidence compared with female residents (procedural male $6.9(1.3)$ vs. female $6.5(1.3), p=$ 0.09 or non-procedural male 6.6 (1.6) vs. female $6.0(1.5), p=$ 0.03 ) (Table 2). Female residents going into GIM demonstrated higher reported confidence than male residents planning GIM careers, though this was not statistically significant (male 5.8 (1.5) vs. female $6.5(1.5), p=0.08$ ) (Fig. 1). The mean confidence rating of female residents going into GIM (6.5 (1.5)) was about the same as the mean confidence rating of female residents going into a procedural specialty $(6.5(1.3))$ (Table 3).

Male residents rated "desire to perform procedures" and "earning potential" as having significantly higher influence in their career decision than female residents ( $p=0.04$ and $p=$ 0.03 , respectively). No other factors significantly differed in importance between genders (Table 4).

One hundred and two residents (34.9\%) reported no debt, 109 (37.3\%) reported \$1-199,999 in debt, and 81 $(27.7 \%)$ reported greater than $\$ 200,000$ in debt. There was no correlation between the amount of debt and resident career choice $(p=0.14)$. There was no difference in confidence between institutions (NW 6.5 (1.4), UTSW $6.4(1.5), p=0.31)$.

\section{DISCUSSION}

In our study, male IM residents with higher self-reported confidence were more likely to choose a procedural subspecialty. On the contrary, female IM residents' self-reported confidence was not predictive of career choice. To our knowledge, this is the first study showing this difference. One could speculate that reasons other than self-reported confidence may be more influential drivers of career choice for women. Prior data have shown that women more highly value long-term patient relationships, time for non-work activities, time with family, more stable work hours, and a more family/female friendly work environment. ${ }^{1,7}$ Interestingly, our survey

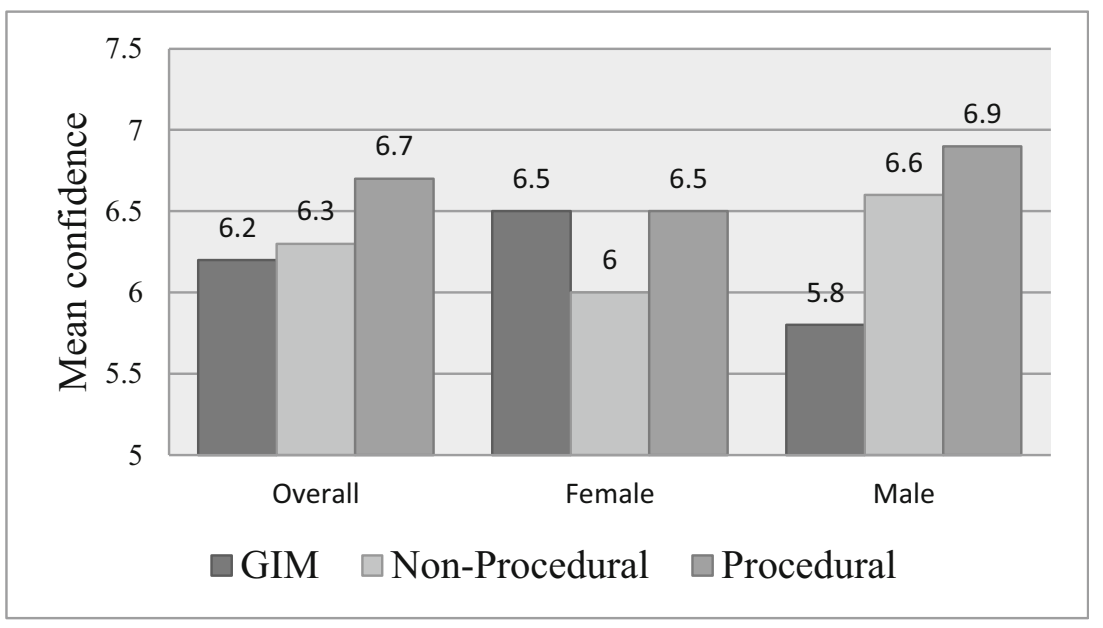

Note: Confidence was measured on a 9-point Likert scale.

Figure 1 Mean confidence by career choice. 
Table 3 Multiple Linear Regression Results for Overall Confidence Score

\begin{tabular}{llll}
\hline \hline & $\boldsymbol{B}$ & SD & $\boldsymbol{p}$ value \\
\hline Female & -0.36 & 0.12 & 0.002 \\
PGY-1 & Reference & & \\
PGY-2 & 1.27 & 0.16 & $<0.001$ \\
PGY-3 & 2.57 & 0.14 & $<0.001$ \\
GIM & Reference & & \\
Non-procedural subspecialty & 0.114 & 0.16 & 0.48 \\
Procedural subspecialty & 0.40 & 0.16 & 0.01 \\
\hline
\end{tabular}

$B$, regression coefficient; $S D$, standard deviation

Dependent variable: confidence score on 9-point Likert scale. Regression coefficients represent the change in confidence score predicted by a given variable

responses showed no gender difference in desire for more time for family or desire for long-term patient relationships.

Regarding other potential reasons for difference in selfreported confidence by gender, we consider the following: Because procedural subspecialties have higher earning potential, a significant influencer on men, these residents may seek out procedures more frequently, resulting in more feedback, and therefore higher confidence. ${ }^{20}$ This is supported by recent literature showing that male residents perform higher numbers of procedures, though more data is needed to rule out selection and reporting bias. ${ }^{21}$ Another consideration is gender differences in response to feedback, leading to differences in confidence. This has been seen in the business literature, where women more quickly aligned their self-ratings with peers' views, resulting in decreased confidence, compared with their male counterparts. ${ }^{22}$ Finally, gender difference in feedback, which has been documented in graduate medical education, is likely another contributing factor. ${ }^{23}$

It is important to note that higher self-reported confidence in residents may not correlate with a higher level of competence. Psychology literature describes the Dunning-Kruger effect, whereby individuals with poor insight into their abilities are associated with high self-reported confidence, despite low competence. ${ }^{18}$ Therefore, it cannot be assumed that male residents' higher self-reported confidence correlates with level of competence. Similarly, it cannot be accurately inferred that higher proportions of men in IM procedural subspecialties is a reflection of a gender difference in competence in procedural skills.
Our study was not designed to investigate the correlation between self-reported confidence and competence in participants.

Male residents reporting higher ratings of self-confidence than female residents, regardless of task, is consistent with prior literature ${ }^{13}$, and in our study, was observed in first-year residents. Therefore, it can be assumed that this divergence occurred prior to residency. The continued difference observed in subsequent years of training suggests one of two things: (1) factors contributing to this difference persisted in the training environments, and/or (2) factors that caused the gender difference prior to start of training, such as gender imprinting, have a legacy effect that persists over time, despite change in environment.

This study is limited by self-reporting confidence, without objective evaluation of skill or ability for correlation. However, self-reported confidence is a meaningful metric, given individual's perception of social norms, perception of self, and perception of others.

Our data is incongruent with prior data on female residents' factors affecting career choice. However, our study represents two large residency programs from different regions of the USA, with near equal representation of men and women, such that our findings may represent changing trends, particularly since our time frame of acquisition differs by over a decade. ${ }^{1,7}$ Additionally, our high study participation better powered the study yield, and the higher number of third-year residents increases the likelihood that indicated career choices are accurate.

Self-reporting is subject to both the respondents being either overly critical or self-inflating of their scores. ${ }^{22}$ Some of this deviation should be mitigated, as the residents' responses were blinded to the research team and were only analyzed in aggregate, and these ratings had no bearing on resident evaluation and training.

The survey used has not been previously validated in another population; however, its validity is supported by its design based on the EPA domains set out by the AAMC, as accepted expectations for graduating IM residents. Additionally, despite the originality of survey design, the responses demonstrated anticipated gains in confidence with time spent in training, supporting survey design validity.

Table 4 Factors Influencing Career Choice

\begin{tabular}{|c|c|c|c|c|}
\hline & Overall $(n=291)$ & Male $(n=145)$ & Female $(n=146)$ & $p$ value \\
\hline Preference for setting of practice (inpatient vs. outpatient) & $3.84(0.82)$ & $3.77(0.81)$ & $3.91(0.82)$ & 0.15 \\
\hline Desire to develop long-term patient relationships & $3.74(1.03)$ & $3.66(1.06)$ & $3.82(0.99)$ & 0.18 \\
\hline Desire for more time for family & $3.62(1.00)$ & $3.56(0.96)$ & $3.68(1.05)$ & 0.28 \\
\hline Desire to work with high acuity patients & $3.46(1.04)$ & $3.51(1.04)$ & $3.40(1.04)$ & 0.39 \\
\hline Current or past mentorship & $3.42(1.15)$ & $3.45(1.23)$ & $3.39(1.06)$ & 0.69 \\
\hline Desire for more time for non-work activities & $3.41(1.04)$ & $3.38(1.00)$ & $3.45(1.07)$ & 0.60 \\
\hline Earning potential & $3.09(0.98)$ & $3.22(0.97)$ & $2.96(0.97)$ & 0.03 \\
\hline Desire to perform procedures & $3.01(1.26)$ & $3.17(1.30)$ & $2.86(1.20)$ & 0.04 \\
\hline Significant other or family influence & $2.77(1.26)$ & $2.71(1.24)$ & $2.83(1.28)$ & 0.43 \\
\hline Administrative responsibilities & $2.41(0.96)$ & $2.36(0.98)$ & $2.47(0.93)$ & 0.35 \\
\hline Personal debt & $2.26(1.16)$ & $2.19(1.16)$ & $2.32(1.17)$ & 0.34 \\
\hline
\end{tabular}

Factor influence on career choice measured on a 5-point Likert scale 
Our study was cross-sectional, limiting the ability to imply causality between factors. A qualitative approach in future work would provide depth, detail, and themes that were not obtainable in our original study design. Career choice was studied at the time of the survey, as opposed to ultimate career choice. This method of acquiring data from a single time point, and from three postgraduate year levels, recognizes that confidence changes over time and may influence career choice throughout. Had we limited data collection to ultimate career choice in senior residents, we would have limited our findings to higher levels of confidence found in these individuals, as observed in our data. A future direction could be to observe a cohort of trainees over time and to include their ultimate career choice. Our study also allowed for selection of only two gender identifiers (male and female), limiting the opportunity for residents to identify as any other self-determined gender. While this study is limited to IM residents, this brings up interesting questions of gender differences in confidence and subspecialty choice in all areas of GME. Further studies in other specialties are needed to determine if this finding crosses into other specialties, as well as into combined program residents such as IM-pediatrics and IMpsychiatry. One limitation of our study was the lack of data in the remaining approximately $20 \%$ of resident non-responders. However, our response rate was high compared with previously published studies $^{1,11}$, and it was necessary to allow residents to opt out of the study for appropriate conduction of research. Finally, there were relatively fewer residents going into GIM, decreasing the power of this sub-group analysis, but our percentage of residents pursuing GIM is comparable to other residencies and prior published reports. ${ }^{24}$

\section{CONCLUSIONS}

Our study reports the novel finding that male IM residents' higher self-reported confidence is associated with a higher likelihood of choosing a procedural subspecialty, as opposed to female IM residents' self-reported confidence, which had no association with career choice. The data from this study further support prior literature showing: (1) men have higher self-reported confidence than women across a variety of clinical tasks, and (2) men report financial incentives as being more influential in career decisions when compared with women. This data is germane to recruitment and diversification of both general IM and its subspecialties. Further insight into understanding the educational, societal, and cultural factors that precipitate these differences, as well as what interventions may modify these outcomes, is needed to encourage diverse representation in all areas of IM.

Acknowledgments: The authors thank Dr. Salahuddin Kazi and Dr. Diane Wayne for their support at the respective institutions.

Corresponding Author: Melanie S. Sulistio, MD; Department of Internal Medicine, Division of Cardiology, University of Texas Southwestern, Dallas, TX, USA (e-mail: Melanie.Sulistio@UTSouthwestern. edu).
Data Availability The datasets analyzed during the current study are available from the corresponding author on reasonable request.

\section{Compliance with Ethical Standards:}

Ethical Approval: Ethical approval has been waived by the institutional review boards (IRB) at both Northwestern University and University of Texas Southwestern

Conflict of Interest: The authors declare that they do not have a conflict of interest.

\section{REFERENCES}

1. Douglas PS, Rzeszut AK, Bairey Merz CN, et al. Career Preferences and Perceptions of Cardiology Among US Internal Medicine Trainees: Factors Influencing Cardiology Career Choice. JAMA Cardiol. 2018;3(8):682-691

2. Douglas PS. Role Models, Allies, and Diversity-Global Issues for Female Cardiology Trainees-Reply. JAMA Cardiol. 2018;3(12):1252.

3. Stone AT, Carlson KM, Douglas PS, Morris KL, Walsh MN. Assessment of Subspecialty Choices of Men and Women in Internal Medicine From 1991 to 2016. JAMA Intern Med. 2019;180(1):140-141.

4. Merrill JM, Camacho Z, Laux LF, Lorimor R, Thornby JI, Vallbona C. Uncertainties and ambiguities: measuring how medical students cope. Med Educ. 1994;28(4):316-322.

5. Geller G, Faden RR, Levine DM. Tolerance for ambiguity among medical students: implications for their selection, training and practice. Soc Sci Med (1982). 1990;31(5):619-624.

6. Chung YC, Lin CY, Huang CN, Yang JH. Perceptions on gender awareness and considerations in career choices of medical students in a medical school in Taiwan. Kaohsiung $\mathrm{J}$ Med Sci. 2013;29(11):629-635.

7. West CP, Drefahl MM, Popkave C, Kolars JC. Internal medicine resident self-report of factors associated with career decisions. J Gen Intern Med. 2009;24(8):946-949.

8. Connelly MT, Sullivan AM, Peters AS, et al. Variation in predictors of primary care career choice by year and stage of training. J Gen Intern Med. 2003; 18(3): 159-169.

9. Burack JH, Irby DM, Carline JD, Ambrozy DM, Ellsbury KE, Stritter FT. A study of medical students' specialty-choice pathways: trying on possible selves. Acad Med. 1997;72(6):534-541.

10. Hauer KE, Durning SJ, Kernan WN, et al. Factors associated with medical students' career choices regarding internal medicine. JAMA. 2008;300(10):1154-1164.

11. McMurray JE, Schwartz MD, Genero NP, Linzer M. The attractiveness of internal medicine: a qualitative analysis of the experiences of female and male medical students. Society of General Internal Medicine Task Force on Career Choice in Internal Medicine. Ann Intern Med. 1993;119(8):812-818

12. Ryan C, Ward E, Jones M. Recruitment and retention of trainee physicians: a retrospective analysis of the motivations and influences on career choice of trainee physicians. QJM. 2018;111(5):313-318.

13. Nomura K, Yano E, Fukui T. Gender differences in clinical confidence: a nationwide survey of resident physicians in Japan. Acad Med. 2010;85(4):647-653

14. Blanch DC, Hall JA, Roter DL, Frankel RM. Medical student gender and issues of confidence. Patient Educ Couns. 2008;72(3):374-381.

15. Blanch-Hartigan D. Medical students' self-assessment of performance: results from three meta-analyses. Patient Educ Couns. 2011;84(1):3-9.

16. Madrazo L, Lee CB, McConnell M, Khamisa $\mathbf{K}$. Self-assessment differences between genders in a low-stakes objective structured clinical examination (OSCE). BMC Res Notes. 2018;11(1):393.

17. Holmboe ES, Huot SJ, Brienza RS, Hawkins RE. The association of faculty and residents' gender on faculty evaluations of internal medicine residents in 16 residencies. Acad Med. 2009;84(3):381-384.

18. Kruger J, Dunning D. Unskilled and unaware of it: how difficulties in recognizing one's own incompetence lead to inflated self-assessments. J Pers Soc Psychol. 1999;77(6):1121-1134.

19. Harris PA TR, Thielke R, Payne J, Gonzalez N, Conde JG. Research electronic data capture (REDCap) - A metadata-driven methodology and 
workflow process for providing translational research informatics support. J Biomed Inform. 2009;42(2):377-381.

20. McCarty PA. Effects of feedback on the self-confidence of men and women. Acad Manag J. 1986;29(4):840-847.

21. Gong D, Winn BJ, Beal CJ, et al. Gender Differences in Case Volume Among Ophthalmology Residents. JAMA Ophthalmol. 2019.

22. Mayo M., Kakarika M, Pastor J.P., Brutus S. Aligning or Inflating Your Leadership Self-Image? A Longitudinal Study of Responses to Peer Feedback in MBA Teams. Acad Manag Learn Educ. 2012;11(4):631-652.
23. Mueller AS, Jenkins TM, Osborne M, Dayal A, O'Connor DM, Arora VM. Gender Differences in Attending Physicians' Feedback to Residents: A Qualitative Analysis. J Grad Med Educ. 2017;9(5):577-585.

24. Dalen JE, Ryan, K.J., Alpert, J.S. Where Have the Generalists Gone? They Became Specialists, Then Subspecialists. Am J Med. 2017;130(7):766-768.

Publisher's Note: Springer Nature remains neutral with regard to jurisdictional claims in published maps and institutional affiliations. 